\title{
Diamond Detectors
}

D.Asner ${ }^{22}$, M.Barbero ${ }^{1}$, V.Bellin ${ }^{\mathrm{i} 2}$, V Belyaev ${ }^{15}$, J-M.Brom $^{10}$, M.Bruzzi $^{5}{ }^{\text {D. D.Chren }}{ }^{23}$, V.Cindro ${ }^{12}$, G.Claus ${ }^{10}$, M.Cristinziani ${ }^{1}$, S Costa ${ }^{2}$, J.Cumalat ${ }^{24}$, R.D'Alessandro ${ }^{6}$, W.de Boer ${ }^{13}$, D.Dobos ${ }^{3}$, I.Dolenc ${ }^{12}$, W.Dulinski ${ }^{10}$, J.Duris ${ }^{20}$, V.Eremin ${ }^{9}$, R.Eusebi ${ }^{7}$, H.Frais-Kölbl ${ }^{4}$, A.Furgeri ${ }^{13}$, C.Galrsapp ${ }^{3}$, K.K.Gan ${ }^{16}$, M.Goffe ${ }^{10}$, J.Goldstein $^{21}$, A.Golubev ${ }^{11}$, A.Gorišek ${ }^{12}$, E.Grigoriev ${ }^{11}$, R.Hall-Wilton ${ }^{3}$, D.Hits ${ }^{17}$, F. Hügging ${ }^{1}$, H.Kagan ${ }^{16}$, R.Kass ${ }^{16}$, G.Kramberger ${ }^{12}$, S.Kuleshov ${ }^{11}$, S.Kwan ${ }^{7}$, S.Lagomarsino ${ }^{6}$, A.La Rosa ${ }^{3}$, A.Lo Giudice ${ }^{18}$, I.Mandić ${ }^{12}$, C.Manfredotti ${ }^{18}$, C.Manfredotti ${ }^{18}$, A.Martemyanov ${ }^{11}$, M.Mathes ${ }^{1}$, D.Menichelli ${ }^{5}$, M.Mikuž ${ }^{12, *}$, M.Mishina ${ }^{7}$, J.Moss ${ }^{16}$, S.Müller ${ }^{13}$, G.Oakham ${ }^{22}$, P.Olivero ${ }^{18}$, G.Parrini ${ }^{6}$, H Pernegger $^{3}$, R.Potenza ${ }^{2}$, K.Randrianarivony ${ }^{22}$, A.Robichaud ${ }^{22}$, S.Roe ${ }^{3}$, S.Schnetzer ${ }^{17}$, T.Schreiner $^{4}$, S.Sciortino ${ }^{6}$, S.Smith ${ }^{16}$, B.Sopko ${ }^{23}$, K.Stenson ${ }^{24}$, R.Stone ${ }^{17}$, C.Sutera ${ }^{2}$, W.Trischuk ${ }^{19}$, J-W.Tsung ${ }^{1}$, C.Tuve ${ }^{2}$, J Velthuis ${ }^{21}$, E.Vittone ${ }^{18}$, S.Wagner $^{24}$, R.Wallny ${ }^{20}$, P.Weilhammer ${ }^{3}$, N.Wermes ${ }^{1}$

${ }^{1}$ Universität Bonn, Germany, ${ }^{2}$ INFN/University of Catania, Italy, ${ }^{3}$ CERN, Geneva, Switzerland,

${ }^{4}$ Fachhochschule fur Wirtschaft und Technik, Wiener Neustadt, Austria, ${ }^{5}$ INFN/University of Florence, Italy, ${ }^{6}$ Department of Energetics/INFN Florence, Italy, ${ }^{7}$ FNAL, Batavia, USA, ${ }^{9}$ Ioffe Institute, St.

Petersburg, Russia, ${ }^{10}$ IPHC, Strasbourg, France, ${ }^{11}$ ITEP, Moscow, Russia, ${ }^{12}$ Jožef Stefan Institute and Physics Department, University of Ljubljana, Ljubljana, Slovenia, ${ }^{13}$ Universität Karlsruhe, Karlsruhe, Germany, ${ }^{15}$ MEPHI Institute, Moscow, Russia, ${ }^{16}$ The Ohio State University, Columbus, OH, USA, ${ }^{17}$ Rutgers University, Piscataway, NJ, USA, ${ }^{18}$ University of Torino, Italy, ${ }^{19}$ University of Toronto, Toronto, Canada, ${ }^{20}$ UCLA, Los Angeles, CA, USA, ${ }^{21}$ University of Bristol, Bristol, UK, ${ }^{22}$ Carleton University, Ottawa, Canada, ${ }^{23}$ Czech Technical University, Prague, Czech Republic, ${ }^{24}$ University of Colorado, Boulder, CO, US.

Speaker, E-mail: Marko.Mikuzeijs.si 


\section{ABSTRACT}

With the first runs of the LHC in 2010 and luminosity upgrades expected from 2016, ATLAS and CMS are planning to upgrade their innermost tracking layers with the ultimate radiation hard technologies. Chemical Vapour Deposition (CVD) diamond has been used extensively in beam conditions monitors as the innermost detectors in the highest radiation areas of BaBar, Belle, CDF and all LHC experiments. This material is now being considered as a sensor material for the innermost layer(s) of the upgraded trackers. Recently the RD42 collaboration constructed, irradiated and tested polycrystalline and singlecrystal CVD diamond sensors to the highest fluences expected at the super-LHC. We present beam test results of chemical vapour deposition diamond up to fluences of $1.8 \times 10^{16} 24 \mathrm{GeV}$ protons $/ \mathrm{cm}^{2}$ illustrating that both polycrystalline and single-crystal chemical vapour deposition diamonds follow a single damage curve. We report preliminary results on polycrystalline diamond from an alternative manufacturer with promising performance.

19th International Workshop on Vertex Detectors - VERTEX 2010

Loch Lomond, Scotland, UK

June $06-112010$ 


\section{Introduction}

Tracking detectors and beam condition monitors of future experiments at the Large Hadron Collider and its upgrades will be situated in radiation environments several orders of magnitude harsher than those of any current detector. At present detectors for tracking close to the interaction region are based on the mature silicon technology, the radiation hardness of which is being extended with innovative processing (3-D) and operation at higher voltages (charge multiplication). Chemical Vapour Deposition (CVD) diamond has a number of properties which make it an attractive material for detector applications. Its large band-gap $(5.5 \mathrm{eV})$ and large displacement energy (42 eV/atom) make it a material that should be inherently radiation tolerant with very low leakage currents and high thermal conductivity, thus ideal for thermal management. CVD diamond is being investigated by the RD42 Collaboration[1] for use very close to the interaction region where the most extreme radiation conditions are expected.

\section{Diamond as Particle Detector}

Diamond, because of its large resistivity, can be operated as a solid state ionization chamber. In Fig. 1, the basic principle of using diamond as a particle detector is shown [2]. A voltage is applied across a layer of diamond a few hundred microns thick. When a charged particle traverses the diamond, atoms in crystal lattice sites are ionized, promoting electrons into the conduction band and leaving holes in the valence band. On average, 36 electron-hole pairs are created in each $\mu \mathrm{m}$ of diamond traversed by a minimum ionizing particle. These charges drift across the diamond in response to the applied electric field producing a detectable electric signal. Since there may be traps in CVD material we often use the charge collection distance $(C C D)$ to characterize the material. $C C D$ corresponds to the average distance the electron-hole pairs move apart. In thin diamond $C C D$ is limited by edge effects, while in thick (thickness $>C C D$ ) diamond $C C D$ approaches the sum of mean free paths of electrons and holes.

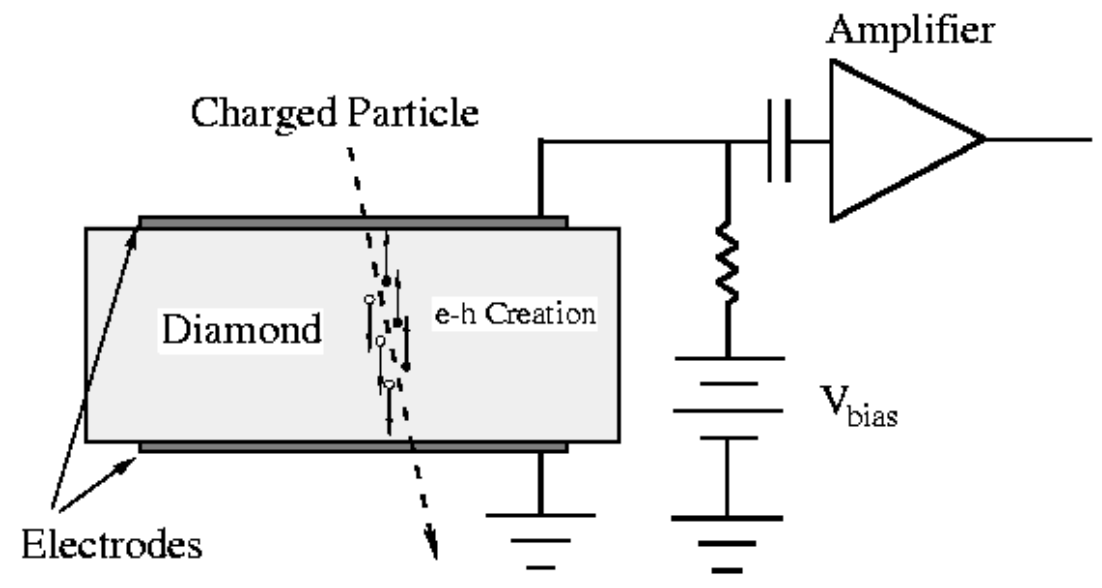

Figure 1: Schematic view of operation of a diamond particle detector. The metal electrodes can be segmented to produce a pixel or strip detector.

There are two flavours of CVD diamond: polycrystalline (pCVD) and single crystal (scCVD). The scCVD is grown on a high-pressure high-temperature diamond substrate 
and forms a perfect diamond lattice. On the other hand, pCVD is grown on a nondiamond substrate, therefore small crystal grains in random orientations start forming on the substrate. The grains grow, and the larger ones have the tendency to grow faster, terminating the growth of the smaller ones. Therefore the average grain size increases across the pCVD thickness from the substrate to the growth side. Also the charge collection properties of the material exhibit improvement with growth thickness. While scCVD exhibits very little trapping, and therefore its $C C D$ matches the detector thickness, $C C D$ of pCVD's is limited by trapping. It is helpful to grow thick pCVD wafers and polish off the substrate side, keeping the high quality material with large grains. Top quality pCVD material today can be grown in 5"wafers, and can exhibit CCD close to $300 \mu \mathrm{m}$ at $500 \mu \mathrm{m}$ thickness and electric field of $2 \mathrm{~V} / \mu \mathrm{m}$. The superb quality of scCVD is for the time being offset by size and price; scCVD, in contrast to 5 "pCVD wafers, is limited to sizes of $1 \mathrm{x} 1 \mathrm{~cm}^{2}$, and the price difference for the small pieces available is nearly an order of magnitude.
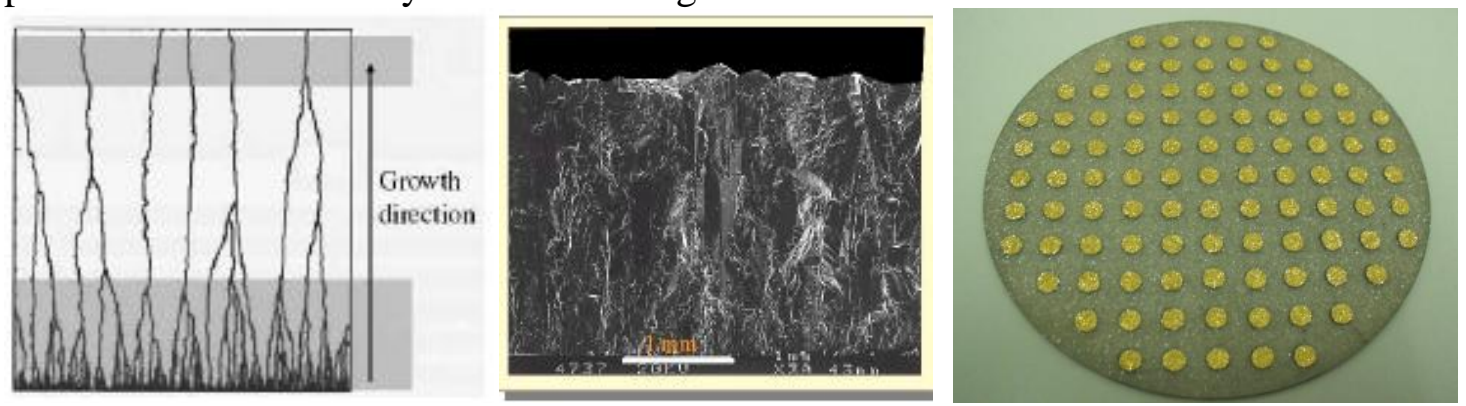

Figure 2: Left to right: schematic growth of pCVD diamond, side view photograph of the diamond (courtesy of Element Six Ltd.), and finished 5 "pCVD diamond wafer with metal test dots on $1 \mathrm{~cm}$ grid.

\section{Radiation Properties of Diamond}

Radiation tolerance might prove as the limiting factor for the utilization of a given sensor material in a high-energy physics experiment at the LHC or its successors. The RD42 irradiation studies consist of characterizing each diamond sample prepared as a tracking detector (strip or pixel) in a high energy test beam before and after each irradiation. The samples prepared as strip detectors are read-out with VA-2 electronics [3] for characterization in the test beam at CERN, whereas the samples prepared as pixel detectors have been so far read-out with ATLAS FE-I3 pixel electronics [4]. All pCVD and scCVD samples were prepared in this manner. Fig. 3 shows a photograph of the metal pattern applied on a diamond strip detector (50 $\mu \mathrm{m}$ pitch), and a complete double sided strip module ready to go into the test beam at CERN. The irradiations described in this paper were performed using $24 \mathrm{GeV}$ protons at the CERN PS facility. The detectors were irradiated at room temperature and in an un-biased state.

Most of the irradiation data were obtained with $24 \mathrm{GeV}$ protons from the CERN Proton Synchrotron, where samples have been taken all the way up to $1.8 \times 10^{16} \mathrm{p} / \mathrm{cm}^{2}$.

Radiation with heavy particles induces displacement damage, resulting in (additional) defects in the crystal lattice. These appear as deep level states in the energy band-gap and act as traps for the traversing charge. Since the band-gap in diamond is large, a trapped charge can remain attached to the trap for months. In principle, a trap, filled with an electron, could trap a hole, resulting in a recombination of a pair, but cross 
sections of neutral traps are an order of magnitude smaller than those of the charged ones. Therefore it is advantageous to expose an irradiated diamond to ionizing radiation and fill the traps - a process known as pumping or priming.
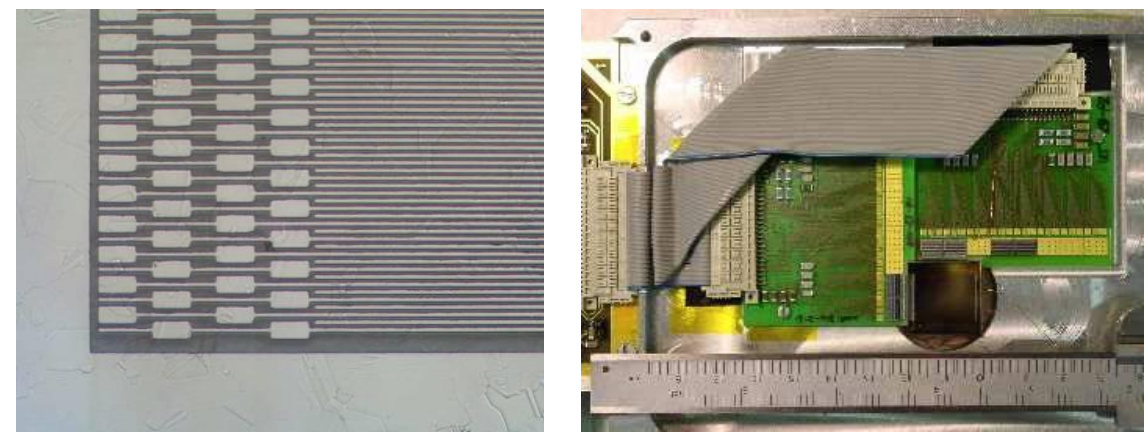

Figure 3: Left: metal strip pattern on diamond, right: double-sided diamond strip module

In the simplest model we assume that additional traps are added to diamond bulk linearly with fluence. As the mean free path is inversely proportional to trap density (and proportional to drift velocity), at fixed electric filed we expect the relation

$$
1 / C C D=1 / C C D_{0}+k \Phi
$$

to describe the degradation of charge collection with radiation fluence. Here, $C C D_{0}$ is the initial charge collection distance, $k$ the damage constant, specific of particle type and energy, and $\Phi$ the particle fluence. Strictly speaking, Eq. (1) is thought adequate for the mean free path, so we are implicitly assuming a thick diamond (thickness $>C C D$ ). Figure 4 exhibits $C C D$ as measured on pCVD and scCVD diamond sensors at a field of $1 \mathrm{~V} / \mu \mathrm{m}$. All sensors were irradiated at the CERN PS. The initial $C C D_{0}$ of pCVD was around $220 \mu \mathrm{m}$. To bring the scCVD sensors onto the pCVD damage curve, with the value of the damage constant $\mathrm{k} \sim 0.7 \times 10^{-18} \mathrm{~cm}^{2} / \mu \mathrm{m}$, their data had to be left-shifted by $3.8 \times 10^{15} \mathrm{p} / \mathrm{cm}^{2}$. This can be viewed as the $24 \mathrm{GeV}$ proton fluence that induces as many traps in the scCVD as are already present in an unirradiated pCVD, or as an effective fluence headroom the scCVD can provide you with respect to off-the-shelf pCVD. There are also two points of a pixel detector added to the plot, due to low fluence their significance is rather low, bur with a somewhat smaller shift $\left(3.2 \times 10^{15} \mathrm{p} / \mathrm{cm}^{2}\right)$ their $C C D$ 's do fall on the same, universal damage curve. With future developments, as $C C D_{0}$ of pCVD diamonds improves, the scCVD damage curve serves also as the ultimate limit of CVD diamond performance under radiation.

The spectrum of hadrons in (s)LHC is predominantly composed of pions in the energy range of several hundred $\mathrm{MeV}$. Therefore, a mechanism is needed to scale the damage between particle species and their energies. The Non Ionizing Energy Loss (NIEL) scaling is widely used in silicon, although departures from NIEL were observed, mainly between charged hadrons and neutrons. For current pCVD diamonds with $C C D_{0}$ in excess of $200 \mu \mathrm{m}$, studies with protons, in addition to $24 \mathrm{GeV}$, are being carried out at energies of 26 (Karlsruhe), 70 (Cyric Facility, Sendai) and $800 \mathrm{MeV}$ (LANL). While the study is still in progress, preliminary data exhibit a significant difference to the published NIEL calculation [5], while better agreement is found with a recent study based on Displacement Per Atom (DPA) as obtained from the new version of FLUKA [6]. A study with reactor neutrons suggests a larger value of the damage constant (3- 
$5 \times 10^{-18} \mathrm{~cm}^{2} / \mu \mathrm{m}$ ) [7], but there the results are plagued by a yet unresolved discrepancy between source and test beam data. With pions, two samples were irradiated up to $6 \times 10^{14} \pi / \mathrm{cm}^{2}$ at PSI [7], but due to the low fluence no precise determination of the damage constant is possible. Although one is tempted to speculate that damage of all charged hadrons would follow the same scaling with energy, a test to relevant fluences with pions would add reassurance to this argument. Using scCVD for this purpose suggests that $\sim 10^{15} \pi / \mathrm{cm}^{2}$ would be sufficient to determine the damage constant with a $10-20 \%$ precision.

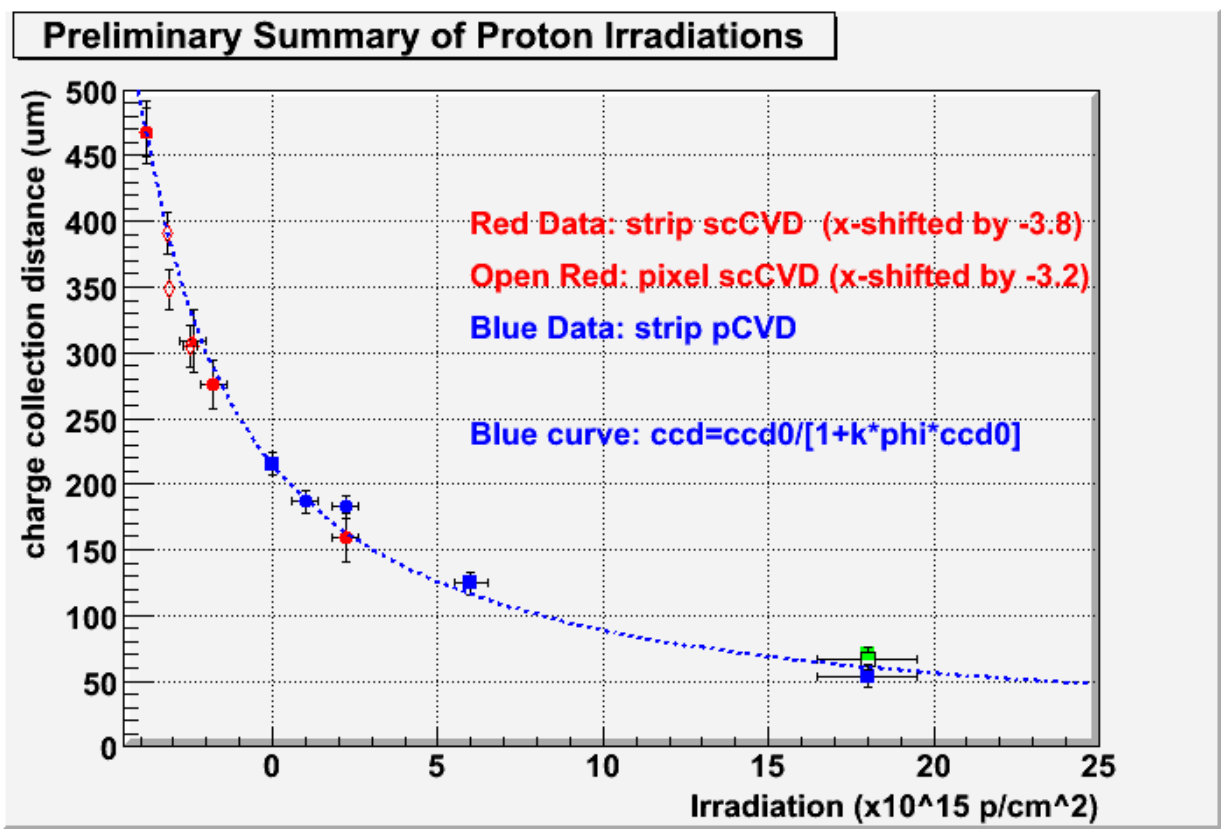

Figure 4: Summary of CVD diamond charge collection data with sensors irradiated with 24 GeV protons at the CERN PS. Both, scCVD and pCVD diamond data fall onto the same damage curve demonstrating a universal mechanism of radiation damage in CVD diamond. All the measurements were taken at an electric field of $E \approx 1 \mathrm{~V} / \mu \mathrm{m}$, except for the green point, taken at $2 \mathrm{~V} / \mu \mathrm{m}$

\section{Application of CVD Diamond in HEP}

CVD diamonds are finding increased application in HEP. Their first usage was for radiation monitoring, acting as solid state ionization chambers. This concept is now used in all LHC experiments; of the order of 100 diamonds are installed in the four detectors.

In addition to monitors operating in current (integration) mode ATLAS and CMS have installed diamond sensors capable of fast (single) particle counting. The ATLAS Beam Conditions Monitor [8] in addition features time-of-flight capability with sub-ns time resolution to resolve beam background from proton collisions.

CMS is building a new luminosity monitor, the Pixel Luminosity Telescope [9], which will employ $48 \mathrm{scCVD}$ diamond sensors, $5 \times 5 \mathrm{~mm}^{2}$ each, in a pointing geometry. This project constitutes the largest deployment of CVD diamond in HEP up to date.

In plans for the tracker upgrade for increased LHC luminosity, both ATLAS and CMS explore the possibility to exploit CVD diamond for the innermost pixel tracker layer(s). As an intermediate, but concrete step towards the upgrade, a group of institutes within ATLAS, gathered around the DPix proposal [10], is bidding with pCVD diamond 
sensors for the Insertable B-Layer [11]. In this scope, several pixel modules have already been built in industry (IZM [12]) with the existing FE-I3 pixel chip, and a qualification programme for the IBL is starting as soon as the new FE-I4 chip becomes available this fall. About 20 modules with pCVD diamond sensors are expected to be built, irradiated and tested by next summer.

\section{CVD Diamond Vendors}

The RD-42 Collaboration has been throughout the years traditionally working with Element 6 [13] and its recent offspring, Diamond Detectors [14] for the supply of electronic grade CVD diamond in both the pCVD and scCVD varieties. With the advent of projects in sight, which require the number of diamond wafers exceeding the number of fingers, the question of multiple suppliers becomes increasingly important, both as a possible price and quality driver, as well as a backup source. RD-42 has explored several companies, specializing in CVD diamond, and promising development has been recently reported with electronic grade pCVD diamond samples obtained from one vendor. These promising results make us eagerly await delivery of HEP-targeted pCVD wafers from this manufacturer. The aspiration is to obtain a close-to-scCVD quality in pCVD format and price.

\section{Conclusions}

Diamond sensors are slowly, but definitely making its way into high energy physics experiments. Currently limited to beam conditions monitors, the ambition is to employ diamond as the ultimate radiation hard material in the innermost tracking layers for the sLHC upgrade. There was considerable improvement of understanding of radiation damage in diamond achieved in the past few years, with a few decisive steps expected to be accomplished in the near future. Industrialization of pixel module production with diamond sensors has been demonstrated by the ATLAS DPix collaboration, and diamonds are in a competitive bid as sensor material for the ATLAS IBL. A very recent development on the vendor market brings hopes to obtain pCVD material with close to scCVD properties.

\section{References}

[1] [CERN/LHCC Reports 97-3, 98-20, 2000-011, 2000-015, 2001-002, 2002-010, 2003-063, 2005003, 2006-010, 2007-002, 2008-005.

[2] S. Zhao, "Characterization of the Electrical Properties of Polycrystalline Diamond Films", Ph.D. Dissertation, Ohio State University (1994).

[3] Integrated Detector and Electronics (IDE) AS designed and manufactures the VA circuits. http://www.ideas.no.

[4] I. Perić, L. Blanquartb, G. Comesa, et al., "The FEI3 readout chip for the ATLAS pixel detector", Nucl. Inst. and Meth. A565(2006)178.

[5] W. de Boer, J. Bol, A. Furgeri, et al., "Radiation hardness of diamond and silicon sensors compared,” Phys. Status Solidi A204(2007)3004-3010.

[6] S. Müller, private communication. 
[7] M.Mikuž, V. Cindro, S. Cline et al., "Study of Polycrystalline and Single Crystal Diamond «Detectors Irradiated with Pions and Neutrons up to $3 \times 10^{15} \mathrm{~cm}^{-2 ،}, 2007$ IEEE Nuclear Science Symposium Conference Record, N44-5.

[8] V. Cindro, D. Dobos, I. Dolenc, et al., “The ATLAS Beam Conditions Monitor”, JINST 3(2008)02004

[9] R. Hall-Wilton, R. Loos, V. Ryjov, et al., "Results from a beam test of a prototype PLT diamond pixel telescope", Nucl. Instr. and Meth. A(2010), doi:10.1016/j.nima.2010.04.097.

[10] H. Kagan, M. Mikuž, W. Trischuk, "Diamond Pixel Modules for the High Luminosity ATLAS Inner Detector Upgrade", ATLAS Upgrade R\&D Proposal, CERN-EDMS ATU-RD-MN-0012.

[11] M. Capeans et al. (ATLAS IBL Collaboration), “ATLAS Insertable B-Layer Technical Design Report”, CERN-LHCC-2010-013 ; ATLAS-TDR-019.

[12] Fraunhofer Gesellschaft, Institut für Zuverlässigkeit und Mikrointegration, High Density Interconnect \& Wafer Level Packaging, Gustav-Meyer-Allee 25, D-13355 Berlin, Germany.

[13] Element Six Ltd, Kings Ride Park, Ascot, Berkshire, SL5 8BP, UK.

[14] Diamond Detectors Ltd, 16 Fleetsbridge Business Centre, Upton Road, Poole, Dorset, BH17 7AF, UK. 